\title{
FRBRização de um catálogo
}

\author{
Fernanda Passini Moreno \\ Universidade de Brasília - UnB, Brasil \\ João Alberto de Oliveira Lima \\ Senado Federal - Brasil
}

CASE REPORTS

\section{Resumo}

O modelo FRBR (Functional Requirements for Bibliographic Records) (IFLA, 1998) vem mudando a forma como lidamos com o catálogo bibliográfico. Uma das principais contribuições desse modelo é a representação da informação dos registros bibliográficos por meio de relacionamentos existentes entre entidades conceituais, Obra e suas Expressões, e físicas, Manifestação e seus Itens. Neste estudo de caso, relatamos a experiência da conversão de 83.257 registros do catálogo da Rede Virtual de Bibliotecas - Congresso Nacional (RVBI), codificados no formato MARC (MAchine Readable Cataloging), para um formato que organiza as informações bibliográficas utilizando os conceitos do modelo FRBR. Como resultado, apresenta-se a informação bibliográfica de forma mais concisa e, consequentemente, gera-se economia de tempo para o usuário no momento da consulta. Além disso, obtivemos resultados que não eram inicialmente esperados, como, por exemplo, a identificação de inconsistências nos registros MARC. A ferramenta "FRBR Display Tool", desenvolvida pela Biblioteca do Congresso Americano, foi fundamental na realização do experimento aqui relatado.

\section{Palavras-chave}

Catalogação ; FRBR ; Representação descritiva

\section{FRBRization of a catalog}

\section{Abstract}

The FRBR model (Functional Requirements it Bibliographic Records) (IFLA, 1998) is changing the way we work with the library catalog. One of the main contributions of this model is the representation of the bibliographic information through relationships between conceptual entities. In this case study we report the experience of converting 83,257 catalog records of the Network Virtual Library - National Congress (RVBI), encoded in the MARC (MAchine Readable Cataloging) to a format that organizes the bibliographic information using the FRBR concepts. As a result, information is presented more concisely literature and consequently generates time savings for the user at the time of the query. Moreover, results obtained were not initially expected, for example, identifying inconsistencies in MARC records. The tool "FRBR Display Tool", developed by the U.S. Library of Congress, was instrumental in the experiment reported here.

\section{Keywords}

Cataloging ; FRBR ; Descriptive representation 


\section{Introdução}

É inegável o impacto do modelo FRBR na organização da informação bibliográfica. Pode ser percebido pela leitura do novo código de catalogação RDA (Resource Description and Access), sucessor do AACR2 (Anglo-American Cataloguing Rules), que incorpora as entidades e os relacionamentos do modelo FRBR, ou ainda pela análise da "Declaração de Princípios Internacionais de Catalogação" (Declaração..., 2009) que posicionou o FRBR, ao lado das "grandes tradições bibliográficas do mundo", como um modelo conceitual fundamental.

Apesar da importância do modelo FRBR, a sua difusão no meio acadêmico e a sua implementação em softwares de gestão de registros bibliográficos avançam em ritmo lento. Pretendemos contribuir para a difusão do modelo FRBR mostrando um exemplo prático da sua aplicação em um grande catálogo. É importante ressaltar que essa não foi a primeira experiência de FRBRização de registros MARC no Brasil. Moreno (2006) aplicou a mesma ferramenta aqui utilizada para FRBRizar 1.584 registros MARC provenientes do catálogo BIBLIODATA da Fundação Getúlio Vargas.

A presente pesquisa caracteriza-se como estudo de caso, notadamente uma pesquisa qualitativa descritiva. Ao examinarmos os registros MARC, utilizamos como critérios os requisitos básicos de dados propostos no nível básico de funcionalidade dos FRBR, como adotados pela ferramenta de conversão "FRBR Display Tool" da Biblioteca do Congresso Americano, utilizada para modelar os registros.

Inicialmente, apresentamos, de forma resumida, as principais entidades do modelo FRBR. Em seguida apresentamos o formato MARC, a linguagem de marcação XML (eXtennsible Markup Language) e o formato MARCXML que combina o conteúdo do registro MARC com a notação XML. Em seguida, apresentamos a ferramenta "FRBR Display Tool" e, ao final, apresentaremos os resultados da FRBRização do catálogo da RVBI.

\section{Modelo FRBR}

Passados quarenta anos da Declaração dos Princípios de Paris (1961), teve início um re-exame de práticas das normas de catalogação, sob a responsabilidade de um grupo de estudos da Seção de Catalogação, Classificação e Indexação da IFLA, materializado no relatório final: FRBR - Functional Requirements for Bibliographic Records: final report, FRBR - Requisitos Funcionais para Registros Bibliográficos, representando um avanço significativo na área de representação bibliográfica. O estudo, iniciado em 1990 e publicado em 1998, reestruturou a forma de exibição dos elementos presentes nos registros bibliográficos, visando satisfazer as necessidades dos diversos usuários que utilizam estes registros.

O modelo FRBR apresenta conceitos de entidades, relacionamentos e atributos, baseados no modelo computacional Entidade-Relacionamento, lançando um novo olhar sobre o objeto bibliográfico, centrado no usuário e suas ações. Tornou-se, ao longo dos últimos anos, objeto de teses, dissertações, grupos de estudos permanentes, para inclusão de melhorias no próprio modelo e em todo o conjunto por eles afetado - normas, formatos e códigos.

Ao propor relacionamentos de diversas naturezas, mais amplos que os hoje existentes nas regras e formatos de uso internacional, os FRBR propõem o agrupamento de entidades com semelhanças em diversos níveis, oferecendo um maior número de opções aos usuários que buscam informações nos registros bibliográficos.

Ao todo, são listadas dez entidades, dividas em três grupos:

- Grupo 1 - entidades que são produto de trabalho intelectual ou artístico;

- Grupo 2 - entidades que são responsáveis pelo conteúdo intelectual, guarda ou disseminação das entidades do primeiro grupo; e

- Grupo 3 - entidades que são ou podem ser assunto das entidades.

As quatro entidades que pertencem ao primeiro grupo compreendem entidades que são produto de trabalho intelectual ou artístico: obra, expressão, manifestação e item. De forma resumida, temos que: 
Obra é uma entidade abstrata, uma criação intelectual ou artística distinta. A entidade Expressão é a realização intelectual ou artística específica que assume uma obra ao ser realizada, excluindo-se aí aspectos de alteração da forma física. Uma Manifestação é a materialização de uma expressão de uma obra, ou seja, seu suporte físico, que podem ser livros, periódicos, kits multimídia, filmes, etc., que é representada pelo Item, um único exemplar de uma manifestação.

As duas últimas entidades refletem a forma física, são entidades concretas, enquanto as duas primeiras refletem o conteúdo intelectual ou artístico.

As definições das entidades do primeiro grupo podem gerar dúvidas quanto a sua natureza, principalmente 0 conceito de obra: por ser demasiado abstrato, e podendo sofrer variações de interpretação de uma cultura para outra, é reconhecida como entidade através de suas expressões.

Portanto, temos como expressões:

- textos variantes (revisões ou atualizações de um texto anterior, i.e., uma obra);

- $\quad$ sínteses $^{1}$, ampliações;

- adição de partes ou acompanhamento a uma composição musical;

- traduções;

- transcrições e arranjos musicais;

- versões dubladas ou versões legendadas;

Quando as alterações representam um "grau significativo de esforço intelectual ou artístico" (IFLA,1998, p. 17), são consideradas novas obras:

- paráfrases, reescritos;

- adaptações para crianças;

- paródias;

- variações musicais sobre um tema;

- transcrições livres de uma composição musical;

- adaptações de uma forma literária ou artística para outra - dramatizações, adaptações de artes gráficas de um meio pra outro;

- resumos, condensações e sumarização ${ }^{2}$

Nota-se que as definições acima esperam abarcar diferentes tipos de criações intelectuais, em qualquer meio, mas que os limites entre uma obra e uma expressão não são realmente claros - sínteses e ampliações são novas expressões, no entanto, condensações, são consideradas novas obras.

As entidades do primeiro grupo serão mais facilmente percebidas nos exemplos que se seguem, a partir de Tillet (2003, p.10) e com a representação hierárquica, na Figura 1 - Exemplos de entidades do Grupo 1, adaptado de Beacom (2003). Também será possível visualizar essas entidades na apresentação dos atributos (subseção 2.1.2, deste capítulo) que por definição, servem para identificar uma entidade. 
Quando alguém se refere a "um livro", está descrevendo um objeto físico, com folhas de papel e demais características que Ihe são próprias. Para os FRBR, isso é um item. Também quando alguém se refere a um livro, pode querer dizer uma "publicação", conhecendo seu ISBN (International Standard Book Number - Número Internacional Normalizado para Livros), inclusive. Para os FRBR, trata-se de uma manifestação. No entanto, caso pense, "um livro, que é tradução de...", tendo em mente um texto em particular, em um idioma específico, para os FRBR trata-se de uma expressão.

Nota-se, na Figura 1, a obra romance literário. Um romance, enquanto obra, pode ser "realizado" de várias maneiras: o texto original, o texto traduzido para outra(s) língua(s) ou uma edição ilustrada, que são as várias expressões de uma obra original.

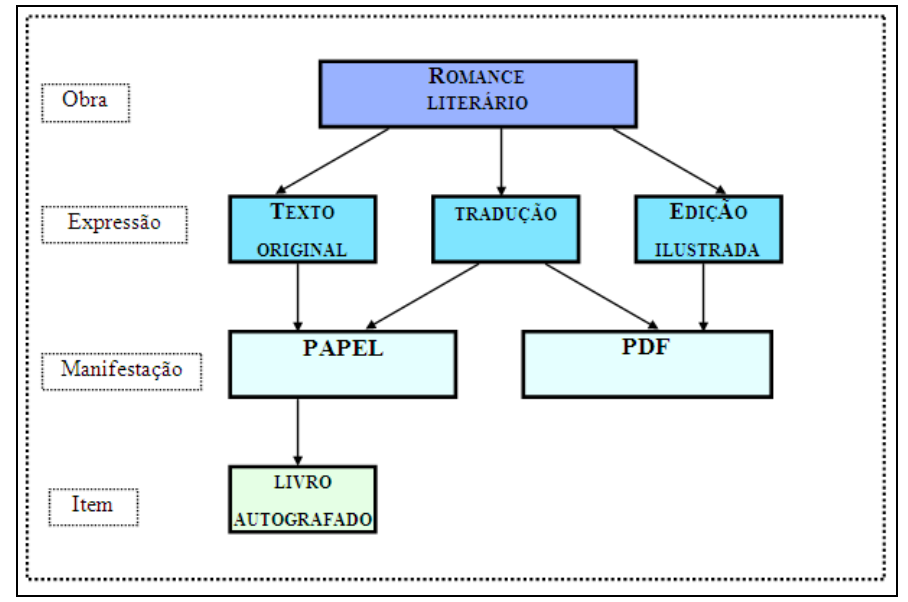

Figura 1. Exemplos de entidades do Grupo 1 Fonte: Moreno (2006), adaptado de Beacom (2003)

A obra, expressa pelo texto original está contida na manifestação em papel, exemplificada pelo livro autografado, sendo que este encadeamento hierárquico reflete as relações bibliográficas primárias.

Qualquer mudança no conteúdo intelectual ou artístico constitui mudança na expressão. Assim, se um texto é revisto ou modificado, a expressão daí resultante é considerada como uma nova expressão, não importando quão aparentemente pequena possa ser a modificação. Nesse exemplo, temos a expressão "tradução" da obra, que "está contida" em papel e como um documento em meio eletrônico, no formato PDF.

A expressão da obra como "edição ilustrada" "está contida" na manifestação como um documento em meio eletrônico no formato PDF. O que caracteriza uma manifestação é o suporte onde a expressão de uma obra é encontrada: seja em meio eletrônico, seja em papel.

\section{Formato MARC}

O formato MARC, desenvolvido durante a década de 1960 pela Library of Congress, tinha como objetivo permitir que esta viabilizasse: "a comunicação de descrições bibliográficas em formato legível por computador, de tal modo que seus registros [da LC] pudessem ser formatados para atender a qualquer objetivo imaginável” (ROWLEY, 1994, p. 77).

O formato MARC, posteriormente chamado de USMARC, passou por muitas modificações desde o projeto inicial. Outros países adaptaram o formato e criaram modelos próprios a partir do MARC. No entanto, as diferenças entre os formatos internacionais aumentaram com o passar do tempo, fazendo-se necessária a criação de um formato internacional. O formato evolui para UNIMARC (Universal MARC) em 1976, pretendendo-se padrão internacional, porém, até hoje os "dialetos" MARC persistem. 
As figuras 2 e 3 apresentam um registro bibliográfico no formato MARC e o mesmo no formato OPAC (On-line Public Access Catalog).

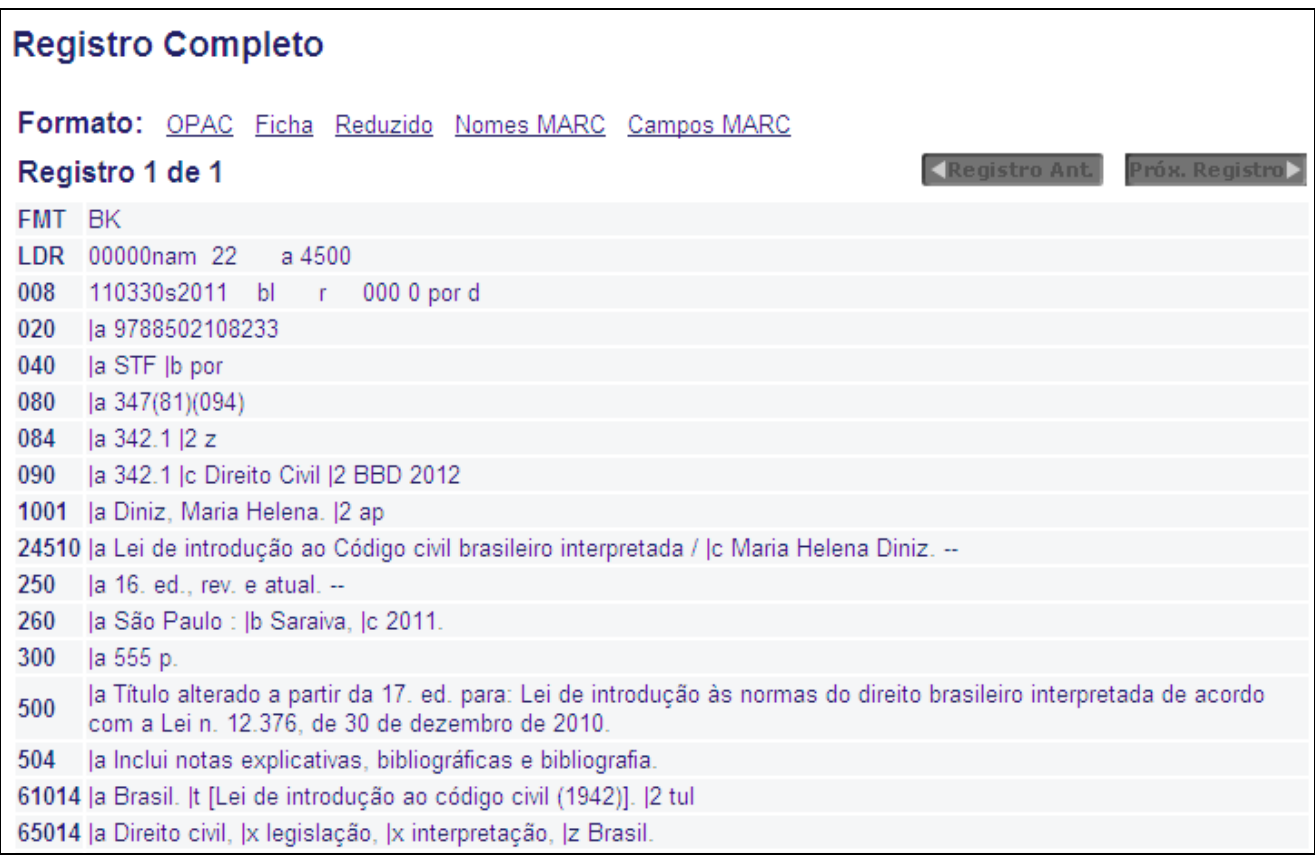

Figura 2. Registro no formato MARC

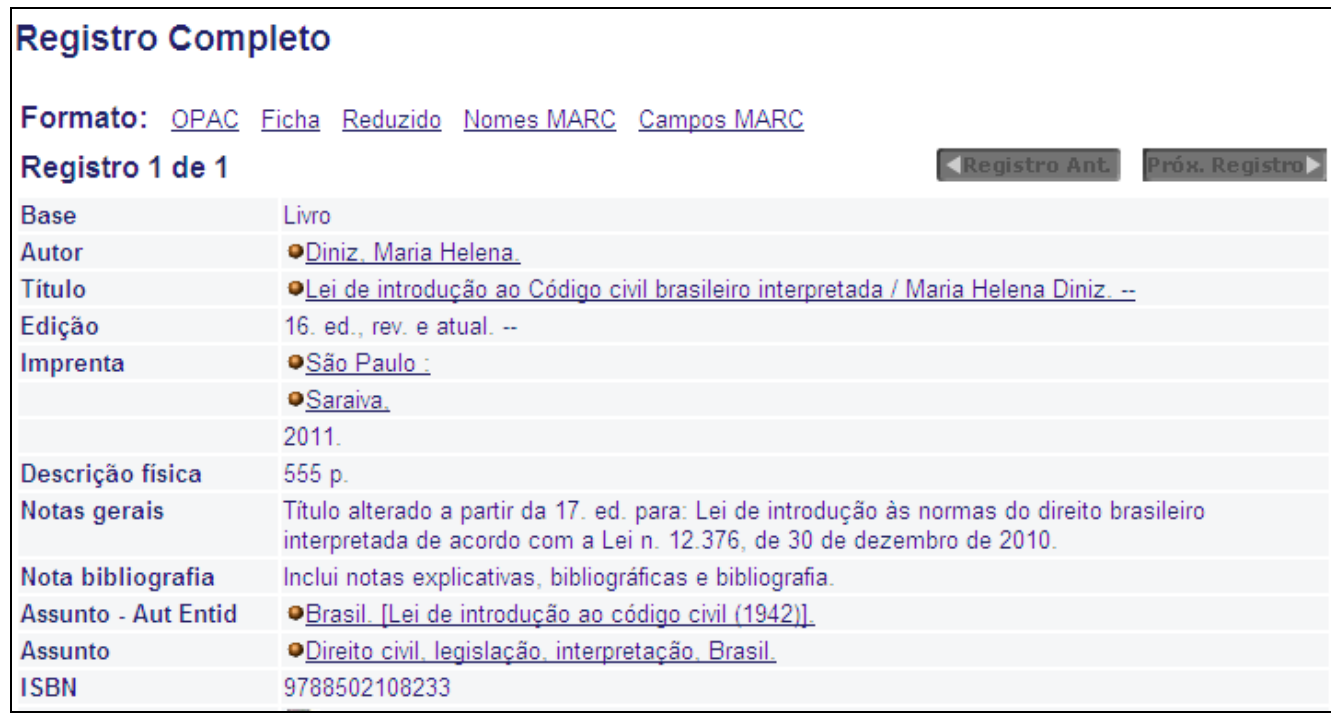

Figura 3. Registro formato OPAC 


\title{
4. Linguagem de marcação XML
}

A linguagem XML (Extensible Markup Language) é derivada de uma linguagem desenvolvida pela IBM na década de 1960, a GML (General Markup Language) que foi a base para o desenvolvimento do padrão ISO 8879 SGML (Standard Generalized Markup Language).

A SGML é uma linguagem para descrever a estrutura dos documentos, ou qualquer tipo de dado textual, deixando a interpretação dos dados para outros programas. Por ser um padrão muito complexo, o W3C (World Wide Web Consortium), consórcio internacional que visa ao desenvolvimento de padrões para WEB, publicou, no final da década de 1990 a XML, que pode ser considerada um "subconjunto" da SGML (FIANDER, 2001; p. 18; W3C, 2005).

Os autores Almeida e Cendon (2003, p.7) comparam as linguagens HTML (Hypertext Markup Language) e XML da seguinte maneira:

\begin{abstract}
A diferença da linguagem XML para a linguagem HTML, atual padrão em uso na Internet, é que as marcações da linguagem XML não são fixas, ou seja, podem ser criadas de acordo com a necessidade do autor. A linguagem HTML foi desenhada para descrever apresentação e a linguagem XML para descrever conteúdo. A linguagem XML permite maior facilidade para interpretação dos dados por computadores, maior facilidade para criação de aplicativos e é um formato livre de relações com fabricantes de software e hardware.
\end{abstract}

Um dos problemas relacionados a HTML é a falta de padronização, que prejudica a interoperabilidade. Os erros gerados na criação de uma página em HTML são interpretados de uma maneira diferente por cada navegador, o que com a linguagem XML não é possível (W3C, 2005).

Bosak (1997) aponta os três principais problemas com o uso da HTML: extensibilidade, estrutura, e validação. Segue ainda dizendo que a XML difere da HTML em três principais aspectos: os provedores de informação podem definir novas tags e atribuir nomes à vontade; a estrutura do documento pode ser "aninhada"3 em algum nível de complexidade; qualquer documento XML pode conter uma descrição opcional de sua gramática para uso em aplicações que necessitam de validação estrutural.

É importante ressaltar que a linguagem XML "foi projetada para: armazenar, transportar e trocar dados. $A$ XML não foi desenvolvida para exibir dados" (W3C, 2005), como é o caso da HTML. No entanto, é necessária uma conversão para HTML para os dados serem lidos pelos navegadores, através do uso de XSL - eXtensible Stylesheet Language, uma linguagem de folha de estilos (ALMEIDA e CENDON, 2003, p. 9). Segundo o tutorial do W3C (2005), uma das aplicações da XSL é transformar XML em HTML antes de ser mostrada ao navegador. Segundo a recomendação do W3C (1999, tradução livre), "a XSLT (XSL Transformations), foi projetada para uso como parte da XSL. A XSL inclui um vocabulário de XML para especificar a formatação. A XSL especifica o estilo de um documento XML usando XSLT para descrever como o documento é transformado em outro documento XML (...)" ou HTML.

\section{Formato MARCXML}

Siqueira e Santos (2004) elaboraram elucidativo capítulo sobre a versão XML do formato MARC21, relatando mais um dos esforços da LC para atualizar e prolongar o uso do formato. Nesta iniciativa, a LC (2005b) propaga que:

[...] está desenvolvendo uma estrutura para trabalhar com dados do MARC em um ambiente de XML. Esta estrutura pretende ser flexível e extensível para permitir que os usuários trabalhem com dados do MARC de maneira específicas a suas necessidades. A estrutura inclui muitos componentes tais como schemas, stylesheets, e softwares.

Os mesmos autores acima citados (p. 106) relatam que:

A estrutura do MARC 21 em XML suporta todos os dados codificados na forma tradicional e sua arquitetura é baseada em componentes, e é extensível, ou seja, permite que os programadores de computador distribuam partes diferentes do software para construir soluções feitas sob encomenda. 
Para realizar essa empreitada, a LC criou uma série de tecnologias adicionais, acima citadas: schemas, stylesheets, e softwares ou ferramentas de conversão. Vejamos alguns exemplos.

Na década de 1990, a LC desenvolveu duas DTDs para SGML, depois convertidas em DTDs para XML, tendo o essencial permanecido para o MARCXML. "Essas DTDs diferem do MARCXML Schema por especificarem cada elemento de dados do MARC como um elemento de XML. Esta aproximação resulta em DTDs muito extensas."(LC, 2005b).

Além disso, a Network Development and MARC Standards Office, gerou um outro schema, chamado MODS (Metadata Object Description Schema). A descrição é feita a seguir, a partir de LC (2005c):

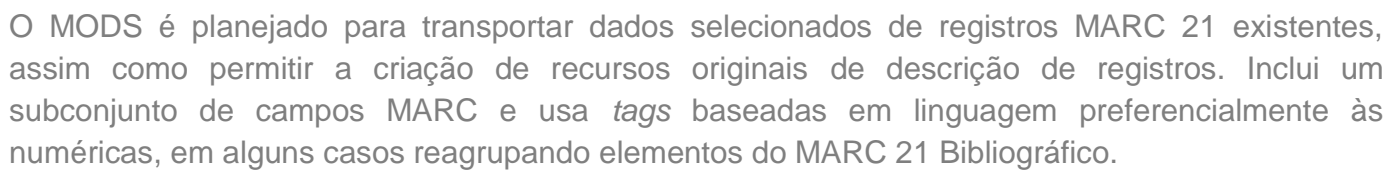

Como ferramentas de conversão, a LC disponibiliza uma variedade de stylesheets (folhas de estilo), além da conversão do formato MARC21 para MARCXML. Há mapeamentos do MARC21 para outros padrões, e conversões reversas também. Podemos citar (a partir de LC, 2005b) como ferramentas de conversão de MARC para: MODS; Dublin Core; OAI MARC para MARC XML; ONIX; MARCXML para MARC DTD Stylesheet (nas versões bibliográfica, autoridade e mista); MARCXML HTML Stylesheet; validação de folhas de estilo MARCXML e MARC Conversion Utility Stylesheet. ${ }^{4}$

\section{Ferramenta "FRBR DISPLAY TOOL"}

A descrição da ferramenta "FRBR Display Tool”, a seguir, é feita parcialmente a partir da tradução de LC (2004).

A ferramenta FRBR Display Tool (versão 2.0) trabalha com arquivos hierarquizados de unidades de registros MARC. Através de sucessivas etapas de transformação, valendo-se de XSLT, re-arranja registros em formato MARC, qualquer que seja o display original, para uma modelagem baseada nos FRBR, de maneira hierárquica para as entidades nos níveis obra, expressão e manifestação. Ressaltamos que a ferramenta não permite uma busca nos catálogos para criar um grupo de registros enquanto opera. Um arquivo de grupos de registros MARC recuperados deve ser criado após o uso da ferramenta.

A ferramenta conversora considera o nível básico de funcionalidade apresentado nos FRBR, que contém os requisitos básicos de dados para bibliografias nacionais, como critérios para seleção dos campos e subcampos a serem investigados em registros MARC e modelagem destes de acordo com os FRBR.

Considerando a proposta do nível básico de dados aliada à análise funcional de Delsey (2002) como norteadores, a ferramenta gera o documento que contém os registros modelados como nos FRBR, através da análise dos seguintes campos e subcampos, resumidamente: 


\section{Nível Obra}

Autor:

- Combinar: os seguintes campos nessa ordem: 100\$a \$b\$c\$d (ou) 110\$a \$b\$c\$d (ou) $111 \$ a \mathrm{ac} \$ \mathrm{~d} \$ \mathrm{n} \$ \mathrm{q}$

- Display: Os seguintes campos nessa ordem: $100 \$ a \$ b \$ c \$ d \$ q$ (ou) $110 \$ a \$ b \$ c \$ d$ (ou) $111 \$ a \$ c \$ d \$ n \$ q$

e Título:

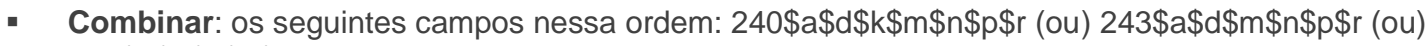
$245 \$ a \$ g \$ k \$ n$

- Exibir: Os seguintes campos nessa ordem: $240 \$ a$ d $\$ k \$ m \$ n \$ p \$ r$ (ou) $243 \$ a$ d $\$ m \$ n \$ p \$ r$ (ou) $245 \$ a \$ g \$ k \$ n \$ p$

Nível Expressão

- Combinar: informações do Líder, posição 06 e campo 008/posições 35-37

\section{Nível Manifestação}

- Combinar: Não há o passo "combinar" neste nível

- Exibir.

- Edição: $250 \$ a \$ b$

- Título: $245 \$ a \$ b \$ n \$ p$ (se o subcampo $\$ b$ começar com um parêntese, não exibir)

- Indicação de responsabilidade: $245 \$ c$

- Imprenta: $260 \$ b \$ c \$ g$

- Descrição física: 300 (todos os subcampos exceto $\$ 6, \$ 8$ )

- ISBN: 020\$a\$c\$z

- ISSN: 022\$a\$yz

- Número do editor (para música): 028\$a\$b

- Reprodução: 533 (todos os subcampos exceto $\$ 6, \$ 7, \$ 8$ )

A conversão dos registros MARC para o formato MARCXML é realizada pelo programa marc4j.jar, que é distribuído juntamente com a ferramenta FRBR Display Tool. Os demais passos são transformações XSLT, detalhadas a seguir:

- Clean.xsl - o primeiro permite filtrar registros e campos que não devem fazer parte da conversão;

- Match.xsl - no segundo passo, são criados elementos nos níveis obra e expressão para serem utilizados no passo seguinte;

- FRBRize.xsl - no terceiro e mais importante passo, os registros são aglutinados de acordo com os elementos da hierarquia FRBR. Internamente, ocorre uma ordenação por Autor, Título. Qualquer erro na cadastramento de Autor e Título, impedirá a correta aglutinação das manifestações em expressões e obras;

- FRBR2HTML.xsI - esse passo, que formato os registros para HTML, no caso do presente estudo, não foi executado. Em seu lugar, criamos um programa de carga dos registros FRBRizados para 0 portal LexML.

\section{FRBRização do catálogo da Rede Virtual de Bibliotecas - RVBI}

O trabalho de FRBRização do catálogo da rede cooperativa de bibliotecas RVBI, coordenada pela Biblioteca do Senado Federal, foi desenvolvido no contexto do Projeto LexML (www.lexml.gov.br). O projeto é uma Rede de Informação Legislativa e Jurídica, o que influenciou a definição da amostra e algumas alterações na rotina padrão, apresentada a seguir. 


\section{Universo e Amostra}

Selecionamos os livros de autores pessoais que contivessem pelo menos a classificação iniciada por "34" (Direito) nos campos 080,082 ou 084 . Esse critério selecionou 83.257 registros MARC. O processamento agrupou 310.415 itens em 83.257 manifestações que foram agrupadas em 63.558 obras. Neste estudo de caso, a quantidade de expressões se igualou ao número de obras, pois não foi realizado o agrupamento das traduções de uma obra. $A$ RVBI registra a informação do título original de uma tradução de forma não padronizada, em um campo MARC diferente do utilizado pela ferramenta FRBR Display Tool.

\section{Resultados}

Além da visualização dos registros bibliográficos no portal da Rede LexML utilizando a hierarquia do modelo FRBR, que será apresentada a seguir, o processo de integração da doutrina permitiu:

- a navegação bidirecional entre os registros bibliográficos e os de normas jurídicas;

- a navegação pela classificação bibliográfica; e

- a identificação de casos de erros de cadastramento de campos MARC.

Relatórios de consistência foram gerados, o que permite a correção dos registros no sistema de gestão bibliográfica, melhorando assim, a cada processamento, a qualidade dos registros bibliográficos.

A Figura 4 apresenta um exemplo da interface do Portal LexML onde é possível perceber como os atributos de Obra, Expressão, Manifestação e Item são apresentados ao usuário. Percebem-se ainda, na parte superior, os links para realização de pesquisa por autor e por classificação. Note-se que toda a hierarquia de classificação é apresentada com links em cada código.

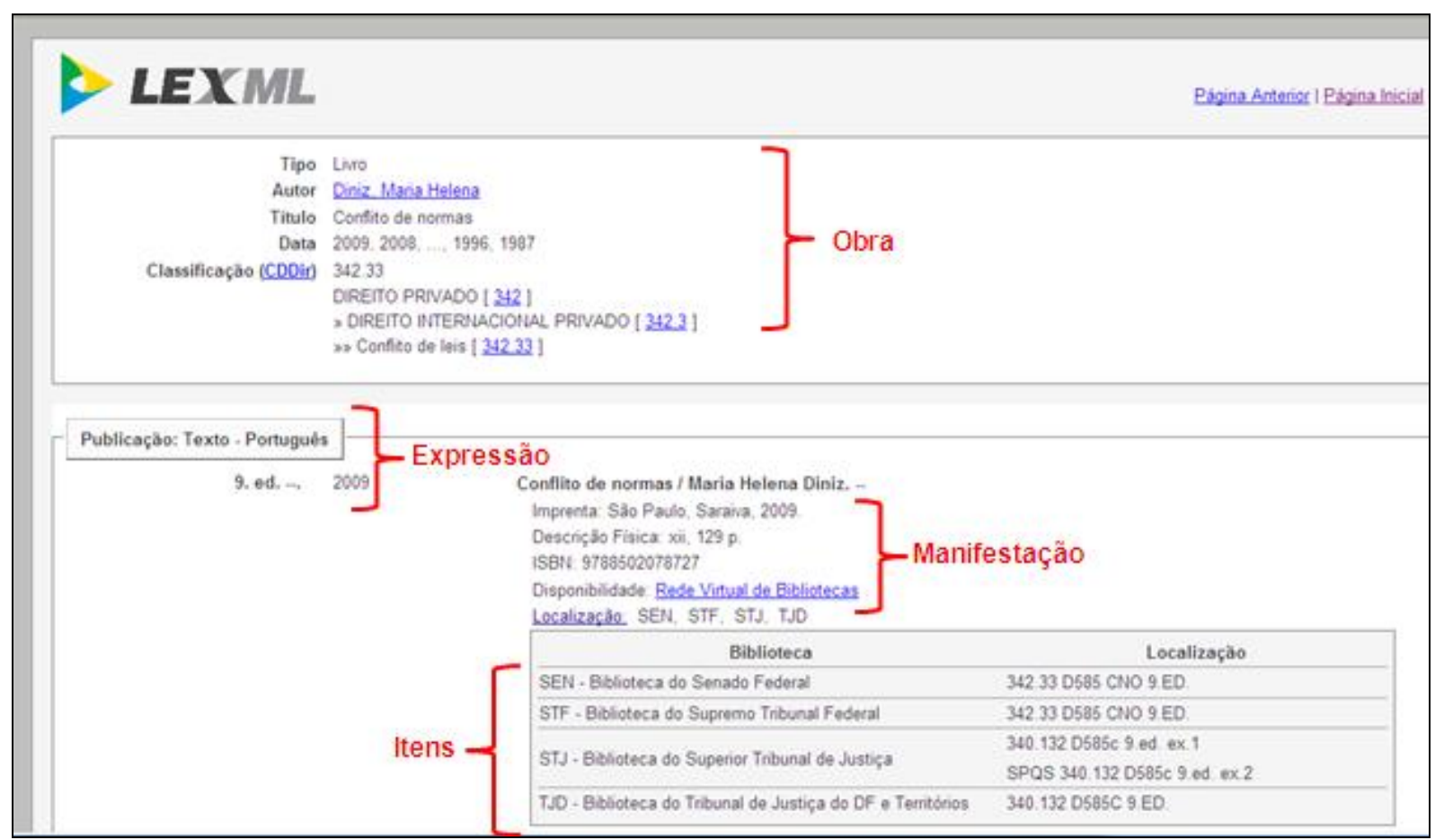

Figura 4. Atributos de Obra, Expressão, Manifestação e Item no Portal LexML. 
$\mathrm{Na}$ visualização proposta, todas as edições de uma obra são apresentadas em uma única página. Por exemplo, a Figura 5 apresenta três edições da obra "Conflito de Normas" de Maria Helena Diniz e, em destaque os links de navegação para normas jurídicas que foram gerados a partir de informações do campo 856 do MARC.

\begin{tabular}{|c|c|c|}
\hline Publicaçào: Texto - Portugués & & \\
\hline 9. ed. - & 2009 & 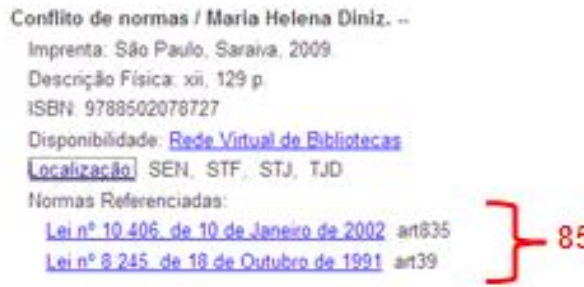 \\
\hline $\begin{array}{l}\text { 8. ed., rev, e atual., de acordo } \\
\text { com o novo Código Civil Lei n. } \\
10.406 / 2008 \text {. }\end{array}$ & 2008 & 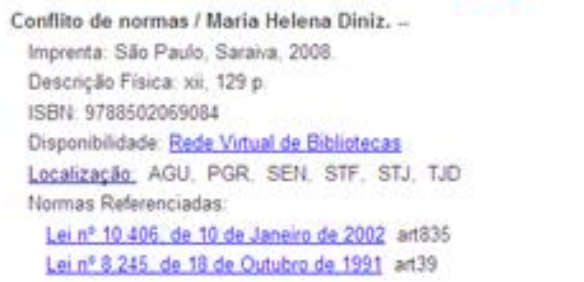 \\
\hline $\begin{array}{l}\text { 7. ed., atual. de acordo corn o } \\
\text { novo Código Civili Loi n. } \\
\text { 10.406/2002. }\end{array}$ & 2007 & 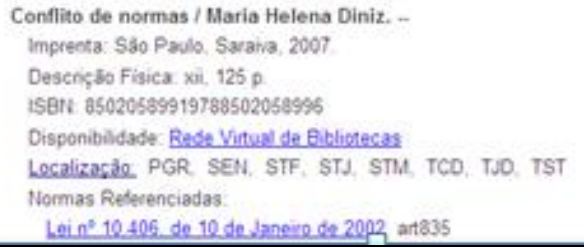 \\
\hline
\end{tabular}

Figura 5. Múltiplas edições em uma única página e links para legislação.

\begin{tabular}{|c|c|c|c|c|c|}
\hline \multicolumn{2}{|c|}{ Doutrina Referenciada } & \multirow[b]{3}{*}{ Titulo } & \multirow[b]{3}{*}{ Responsabilidade } & \multirow[b]{3}{*}{ Classe of } & \multirow[b]{3}{*}{ Dispo } \\
\hline Filtrar & & & & & \\
\hline Edigha * & 1"Autor 4 & & & & \\
\hline 2011 & Borges, Vielson & 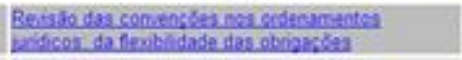 & Nelson Berges - & & at19 \\
\hline 2011 & Borges, tielson & 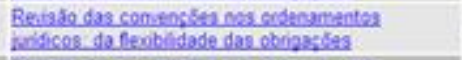 & Nelson Borges .. & & $a+68$ \\
\hline 2011 & Borgeo, Nelsan & 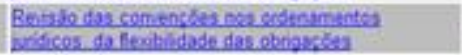 & Nelson Borges. - & & at 69 \\
\hline 2011 & Borges, Naison & 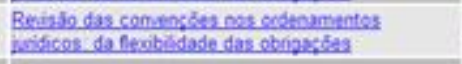 & Nielson Borges: -. & & anto \\
\hline 2011 & $\begin{array}{l}\text { Fucci. Paulo } \\
\text { Eduarda }\end{array}$ & Beforma de ies de inquinate & Paulo Eduardo Fucei - & 34216533 & \\
\hline 2011 & $\begin{array}{l}\text { Male, Calos } \\
\text { Aberto Dabus }\end{array}$ & 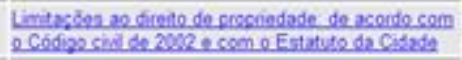 & $\begin{array}{l}\text { Carlos Aberto Dabus Mald : } \\
\text { preficio. Moguel Reale - }\end{array}$ & 342.123 & \\
\hline 2010,2002 & $\begin{array}{l}\text { Cassettari, } \\
\text { Christiano }\end{array}$ & Wulla contratual thens es surica & Christuno Csssettsin - & 342.1422 & \\
\hline 2010 & $\begin{array}{l}\text { Borjes, Isabel } \\
\text { Cristina Pode }\end{array}$ & $\begin{array}{l}\text { Aberacles na lei de ingulinate somentiogralej } \\
1211209\end{array}$ & Isabel Cristina Ponte Berjes - & -342.4533 & \\
\hline 2010 & $\begin{array}{l}\text { Clamara. } \\
\text { Alecandre } \\
\text { Freitas }\end{array}$ & Comentarios a reforma da Leide iocaskes & Alexcandre Freitas Cámara - - & 342,14533 & \\
\hline 2010 & $\begin{array}{l}\text { Frederica. } \\
\text { Alencar }\end{array}$ & Comentainas as atreracles da Le do inquilinate & Alencar Fredenco - & & \\
\hline 2010 & $\begin{array}{l}\text { Gasparetto, } \\
\text { Evelyn Raberta }\end{array}$ & 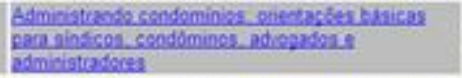 & $\begin{array}{l}\text { Evelyn Roberta Gasparetto, } \\
\text { Cristina Muccio Guidon. - }\end{array}$ & & \\
\hline 2010 & $\begin{array}{l}\text { Giancali Brunno } \\
\text { Panded }\end{array}$ & 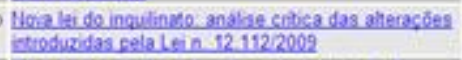 & $\begin{array}{l}\text { Brunno Pandsen Giancoll e } \\
\text { Fabio Vieira Figueinedo - }\end{array}$ & 342.14533 & \\
\hline 2010 & Hanada Fabio & 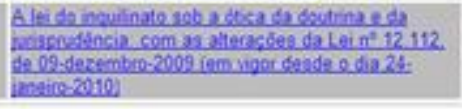 & $\begin{array}{l}\text { Fabio Hanada, Andréa Ranien } \\
\text { Hanada colaberador. Marcelo: } \\
\text { Victor Abbud - }\end{array}$ & 342.14533 & \\
\hline
\end{tabular}

Figura 6. Seção "Doutrina Referenciada" da Lei do Inquilinato. 
A navegação da legislação para a doutrina é realizada por meio de links na seção "Doutrina Referenciada" de cada norma jurídica. A Figura 6 apresenta a doutrina referenciada da Lei do Inquilinato (Lei 8245/1991). Note-se na interface a possibilidade de ordenação e filtro pelo conteúdo de qualquer das colunas.

Como relatado anteriormente, o processo de conversão revelou erros de codificação nos registros MARC. O processo de integração com o LexML gera relatório de erros para correção. A Tabela 1 apresenta alguns tipos de erro do relatório gerado em outubro de 2011.

\begin{tabular}{|l|r|}
\hline \multicolumn{1}{|c|}{ Tipo do Erro } & \multicolumn{1}{|c|}{ Quantidade } \\
\hline Indicador 1 inválido & 7 \\
\hline Indicador 2 inválido & 3652 \\
\hline Idioma inválido & 3 \\
\hline Campo 240 não pode coexistir com 130 & 4 \\
\hline Campo 240 exige presença de 100, 110,111 & 753 \\
\hline Ocorrência de dois campos 260 & 38 \\
\hline Código subcampo inválido & 3 \\
\hline
\end{tabular}

Tabela 1. Erros de codificação MARC identificados no processo de FRBRização.

\section{Conclusão}

Passados quase 15 anos do lançamento do modelo FRBR, percebe-se que a sua adoção ainda é lenta por vários motivos. Apesar da lentidão, percebe-se que o modelo FRBR vem se consolidando e se expandindo. Nos últimos cinco anos, a IFLA publicou os modelos Functional Requirements for Authority Data (FRAD) - e Functional Requirements for Subject Authority Data (FRSAD) que detalham as entidades dos Grupos 2 e 3, respectivamente. No Brasil, ainda existe pouca pesquisa e divulgação do modelo FRBR.

As entidades do modelo FRBR, como descritas, refletem uma nova percepção sobre o objeto bibliográfico: ao distinguir o conteúdo da forma física, e relacioná-los, agrupam-se entidades com semelhanças, porém, de forma mais abrangente do que as opções que um catálogo comum (mesmo que eletrônico) pode vir a oferecer.

O nosso estudo de caso mostrou que, além das vantagens de se ter a informação bibliográfica apresentada de forma mais concisa, um processo de FRBRização pode contribuir com a melhoria da qualidade de codificação dos registros bibliográficos. Por fim, é importante ressaltar que o processo de FRBRização exige uma equipe multidisciplinar pois, invariavelmente, é necessário realizar ajustes que exigem conhecimentos do formato MARC, da linguagem de marcação XML e da linguagem de transformação XSLT.

O resultado deste trabalho, isto é, registros bibliográficos FRBRizados, está disponível para consulta no portal da Rede de Informação Legislativa e Jurídica - LexML (www.lexml.gov.br). O trabalho de FRBRização da RVBI ainda está em curso e deve considerar, nos próximos passos, capítulos de livro e artigos de revista. 


\section{Referencias}

ALMEIDA, Mauricio B.; CENDON, Beatriz V. Pesquisa sobre ferramentas de conversão de registros catalográficos padrão MARC para a linguagem XML. In: ENCONTRO NACIONAL DE PESQUISA EM CIÊNCIA DA INFORMAÇÃO, 5, 2003, Belo Horizonte. Anais. Belo Horizonte: UFM. Escola da Ciência da Informação, 2003. ENAN043. V Encontro Nacional de Pesquisa em Ciência da Informação (ENANCIB), Belo Horizonte (Brasil) 10 de Novembro de 2003.(CD-ROM).

BEACOM, Matthew. The once \& future catalog: the FRBR model, users and catalogs. Out. 2003.Disponível em: <http://www.library.yale.edu/ mbeacom/talk/Once\%20and\%20Future\%20Catalog2.ppt> Acesso em: 02 nov. 2004.

BOSAK, Jon. XML, Java, and the future of the Web. (1997). Disponível em: <http://www.ibiblio.org/pub/suninfo/standards/xml/why/xmlapps. htm>. Acesso em: 05 nov. 2005.

DECLARAÇÃO de princípios internacionais de catalogação. Trad. de Lídia Alvarenga e Márcia Milton Vianna. IFLA, 2009. Disponível em: <http://www.ifla.org/files/cataloguing/icp/icp 2009-pt.pdf>. Acesso em: 4 jul. 2010.

DECLARACIÓN de Principios Adoptados por la Conferencia Internacional sobre Principios de Catalogación París, Octubre de 1961. Traducción: Elena Escolano Rodríguez. In: 2ª . Reunión IFLAde Expertos sobre un Código Internacional de Catalogación. 17 e 18 agosto de 2004, Buenos Aires, Argentina. Disponível em: <http://www.loc.gov/loc/ifla/imeicc/papers-sp.html>. Acesso em: 13 out. 2004.

DELSEY, Tom. Functional analysis of the MARC 21 bibliographic and holdings formats. Washington: Library of Congress, 4 jan. 2002. Disponível em: <http://www.loc.gov/marc/marc-functional-analysis/functional-analysis.html> Acesso em: 10 fev. 2005.

FIANDER, D. Applying XML to the Bibliographic Description. Cataloging \& Classification Quartely, New York, v.33, n.2, p. 17-28, 2001.

IFLA Study Group on the Functional Requirements for Bibliographic Records. Functional requirements for bible/ographic records: final report. Vol. 19, UBCIM Publications - New Series. München: K. G. Saur, 1998.

LIBRARY OF CONGRESS (LC). FRBR Display Tool version 2.0 (2004). Disponível em: <http://www.loc.gov/marc/marc-functionalanalysis/tool.html>. Acesso em: 25 out. 2005.

MARC 21 XML Schema Official Web Site (2005a). Disponível em:

<http://www.loc.gov/standards/marcxml/>. Acesso em: 25 out. 2005.

MODS Metadata Object Description Schema Official Web Site (2005b). Disponível em:

<http://www.loc.gov/standards/mods/>. Acesso em: 25 out. 2005.

MORENO, F. P. Requisitos funcionais para registro bibliográficos - FRBR: um estudo no catálogo da Rede Bibliodata. Brasília, 2006. 199 f. (Mestrado em Ciência da Informação), Programa de Pós-Graduação em Ciência da Informação, Universidade de Brasília, 2006. Disponível em: < http://hdl.handle.net/10482/2565>. Acessado em: 05 jan. 2007.

ROWLEY, J. Informática para bibliotecas. Tradução de Antônio Agenor Briquet de Lemos. 3. ed. Brasília : Briquet de Lemos/Livros, 1994.

SIQUEIRA, Marcos A.; SANTOS, Plácida L.V. A. da C. A versão em XML do MARC 21 e as formas de representação descritiva na Ciência da Informação. In: Tecnologia e conteúdos informacionais: abordagens teóricas e práticas (org. Silvana Ap. G. Vidotti). São Paulo: Polis, 2004. p. 95-111.

TILLETT, Barbara. FRBR: Functional requirements for bibliographic records. Technicalities, vol. 23, n. 5 set/out 2003, p.1, $10-13$.

W3C - World Wide Web Consortium. XML Tutorial. Disponível em: <http://www.w3schools.com/xml/default.asp>. Acesso em: 30 out. 2005.

XSL Transformations (XSLT). W3C Recommendation 16 Nov. 1999. Disponível em:

<http://www.w3.org/TR/xslt\#section-Introduction>. Acesso em: 30 out. 2005. 


\section{Dados dos autores}

\section{Fernanda Passini Moreno}

Possui graduação em Biblioteconomia e Ciência da Informação pela Universidade Federal de São Carlos (2003). Possui mestrado (2006) e doutorado (2011) em Ciência da Informação pela Universidade de Brasília e é Professora Adjunta (DE) na mesma Universidade. É membro do Representação e Organização da Informação e do Conhecimento (EROIC), cadastrado no Diretório do CNPq e certificado pela Instituição. É vice-coordenadora do GT2 Organização e Representação do Conhecimento da ANCIB (2013-2014). Atuou como professora assistente efetiva na Universidade Federal de Goiás (UFG), com carga horária de 20 hs. Atuou como Consultora do Instituto Brasileiro de Informação em Ciência e Tecnologia, desenvolvendo material didático e ministrando cursos sobre o Sistema de Editoração Eletrônico de Revistas (SEER). Ministrou mini-cursos na área de Representação Descritiva sobre os Requisitos Funcionais para Registros Bibliográficos (FRBR) . Tem experiência na área de Ciência da Informação, com ênfase em Representação Descritiva e Comunicação Científica.

fernandam@unb.br

\section{João Alberto de Oliveira Lima}

Possui graduação em Ciência da Computação pela Universidade Federal da Paraíba (1990), mestrado em Ciência da Computação pela Universidade de Brasília (2000) e doutorado em Ciência da Informação pela Universidade de Brasília (2008) com estágio na Universidade de Bolonha (2007). Desde 1995, trabalha como Analista de Informática Legislativa no Senado Federal onde lidera a implementação do Projeto LexML (http://www.lexml.gov.br). joaolima@senado.gov.br
Recebido - Received
: 2013-01-16
Aceitado - Accepted
: 2013-03-30
(c) EY $_{\text {EY }}$ New articles in this journal are licensed under a Creative Commons Attribution 3.0 United States License.

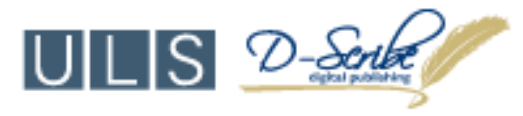

This journal is published by the University Library System of the University of Pittsburgh as part of its D-Scribe Digital Publishing Program and is cosponsored by the University of Pittsburgh Press.

\footnotetext{
1 Abridgements, no original.

2 Abstracts, digests and summaries, no original.

${ }^{3}$ No original: nested. Nest = aninhar. Nesting level = nível de aninhamento = número de sub-rotinas dentro de uma sub-rotina.

${ }^{4}$ OAI - Open Archives Iniciative; ONIX - ONline Information eXchange.

${ }^{5}$ A versão 2.0 da ferramenta FRBR Display Tool está disponível para download em: http://www.loc.gov/marc/marcfunctional-analysis/tool.html (acessado em agosto/2012).
} 